\title{
New Edge Coherent Mode Providing Continuous Transport in Long Pulse H-mode Plasmas
}

Wang, H.Q.; Xu, G.S.; Wan, B.N.; Ding, S.Y.; Guo, H.Y.; Shao, L.M.; Liu, S.C.; Xu, X.Q.; Wang, E.; Yan, Ning

Total number of authors:

27

Published in:

Physical Review Letters

Link to article, DOI:

10.1103/PhysRevLett.112.185004

Publication date:

2014

Document Version

Publisher's PDF, also known as Version of record

Link back to DTU Orbit

Citation (APA):

Wang, H. Q., Xu, G. S., Wan, B. N., Ding, S. Y., Guo, H. Y., Shao, L. M., Liu, S. C., Xu, X. Q., Wang, E., Yan, N., Naulin, V., Nielsen, A. H., Juul Rasmussen, J., Candy, J., Bravenec, R., Sun, Y. W., Shi, T. H., Liang, Y. F., Chen, R., ... Gong, X. Z. (2014). New Edge Coherent Mode Providing Continuous Transport in Long Pulse Hmode Plasmas. Physical Review Letters, 112(18), [185004]. https://doi.org/10.1103/PhysRevLett.112.185004

\section{General rights}

Copyright and moral rights for the publications made accessible in the public portal are retained by the authors and/or other copyright owners and it is a condition of accessing publications that users recognise and abide by the legal requirements associated with these rights.

- Users may download and print one copy of any publication from the public portal for the purpose of private study or research.

- You may not further distribute the material or use it for any profit-making activity or commercial gain

- You may freely distribute the URL identifying the publication in the public portal 


\title{
New Edge Coherent Mode Providing Continuous Transport in Long-Pulse H-mode Plasmas
}

\author{
H. Q. Wang, ${ }^{1}$ G. S. Xu, ${ }^{1,}{ }^{*}$ B. N. Wan, ${ }^{1}$ S. Y. Ding, ${ }^{1}$ H. Y. Guo, ${ }^{1,2}$ L. M. Shao, ${ }^{1}$ S. C. Liu, ${ }^{1}$ X. Q. Xu, ${ }^{3}$ E. Wang, ${ }^{3}$ N. Yan, ${ }^{4}$ \\ V. Naulin, ${ }^{4}$ A. H. Nielsen, ${ }^{4}$ J. Juul Rasmussen, ${ }^{4}$ J. Candy, ${ }^{2}$ R. Bravenec, ${ }^{5}$ Y. W. Sun, ${ }^{1}$ T. H. Shi, ${ }^{1}$ Y. F. Liang, ${ }^{1}$ \\ R. Chen, ${ }^{1}$ W. Zhang, ${ }^{1}$ L. Wang, ${ }^{1}$ L. Chen, ${ }^{1}$ N. Zhao, ${ }^{1}$ Y. L. Li, ${ }^{1}$ Y. L. Liu, ${ }^{1}$ G. H. Hu, ${ }^{1}$ and X. Z. Gong ${ }^{1}$ \\ ${ }^{1}$ Institute of Plasma Physics, Chinese Academy of Sciences, Hefei 230031, China \\ ${ }^{2}$ General Atomics, P.O. Box 85608, San Diego, California 92186-5608, USA \\ ${ }^{3}$ Lawrence Livermore National Laboratory, Livermore, California 94550, USA \\ ${ }^{4}$ Association EURATOM-DTU, Physics Department, DK 2800 Kgs. Lyngby, Denmark \\ ${ }^{5}$ Fourth State Research, 503 Lockhart Drive, Austin, Texas 78704-4335, USA
}

(Received 6 August 2013; published 8 May 2014)

\begin{abstract}
An electrostatic coherent mode near the electron diamagnetic frequency $(20-90 \mathrm{kHz})$ is observed in the steep-gradient pedestal region of long pulse H-mode plasmas in the Experimental Advanced Superconducting Tokamak, using a newly developed dual gas-puff-imaging system and diamond-coated reciprocating probes. The mode propagates in the electron diamagnetic direction in the plasma frame with poloidal wavelength of $\sim 8 \mathrm{~cm}$. The mode drives a significant outflow of particles and heat as measured directly with the probes, thus greatly facilitating long pulse H-mode sustainment. This mode shows the nature of dissipative trapped electron mode, as evidenced by gyrokinetic turbulence simulations.
\end{abstract}

A current bottleneck in magnetic fusion development is the erosion of the plasma-facing wall materials due to transient heat load from edge-localized modes (ELMs). Thus, it is desirable to mitigate ELMs by replacing these transient heat impulses with a benign and continuous transport process, without incurring confinement degradation [1]. In the tokamak high-confinement mode ( $\mathrm{H}$ mode), a transport barrier develops at the plasma edge, where a high-pressure "pedestal" forms. The accumulation of energy and particles inside the pedestal normally leads to the explosive relaxation of the pedestal gradients through ELMs. To exhaust the accumulated energy, fuel particles and impurities in a more moderate and continuous way, an alternative edge mode [2-5] or external control technique [6] is desired. Among them, a good candidate is the trapped electron mode (TEM) [7-10], which is the mode responsible for particle control at the internal transport barrier [9] and density pump out in the central electron heating experiments [10], hence, highly desirable for long-pulse $\mathrm{H}$-mode sustainment, if it can be excited in the $\mathrm{H}$-mode pedestal region.

We have discovered a new edge coherent mode (ECM) near the local electron diamagnetic frequency $(20-90 \mathrm{kHz})$ in the steep-gradient pedestal region, existing continuously throughout a long-pulse $\mathrm{H}$-mode regime with a record pulse length over $30 \mathrm{~s}$, recently achieved in the EAST superconducting tokamak $\left(R_{0}=1.9 \mathrm{~m}, a=0.44 \mathrm{~m}, B_{t}=\right.$ 1.5-2.5 T) [11,12]. This Letter demonstrates, for the first time, that the ECM is able to provide sufficient particle and energy exhaust across the pedestal in $\mathrm{H}$ modes in the absence of ELMs, by direct probing the ECM-driven radial fluxes inside the separatrix using a new diamond-coated reciprocating probe array [13], which allows ELM-less operation in stationary H-mode conditions up to 60 times the energy confinement time [14]. The diamond coating has been demonstrated to be a very effective technique for reducing sputtering and impurity generation from the probe surface, which allows for measurements much deeper into the pedestal. Previous measurements with normal probes are only available up to the separatrix [15]. In addition, the detailed two-dimensional (2D) structure of the ECM in the plane perpendicular to the local magnetic field lines was examined with a unique, newly developed dual gas puff imaging (GPI) system [16], which has two view areas, separated toroidally by $66.6^{\circ}$, up-down symmetrically about the midplane, as shown in Fig. 1(a). Images of the $13 \times 13 \mathrm{~cm}$ square areas with helium gas puff are recorded simultaneously by two fast cameras at a frame rate of $391 \mathrm{kHz}$ and resolution of $64 \times 64$ pixels with 12-bit dynamic range.

The ECM has been observed with the central-lineaveraged density, $\bar{n}_{e}$, varying from 1.9 to $5 \times 10^{19} \mathrm{~m}^{-3}$ $\left(\bar{n}_{e} / n_{G}=0.28-0.7\right)$, corresponding to an electron collisionality, $\nu_{e}^{*}=0.5-5$, evaluated at the top of pedestal. Here, the definition for $\nu_{e}^{*}$ follows Eq. (18b) in [17], $n_{G}=I_{p} / \pi a^{2}$ is the Greenwald density and $I_{p}(0.28-0.6 \mathrm{MA})$ is the plasma current. These $\mathrm{H}$-mode plasmas are achieved in a lowrecycling regime due to extensive lithium wall coating $[11,12]$, with combined lower hybrid current drive (LHCD) and ion cyclotron resonant frequency (ICRF) hydrogen minority heating with central deposition in deuterium plasmas, at a power of $P_{\mathrm{LHCD}}=1-2 \mathrm{MW}$ and 

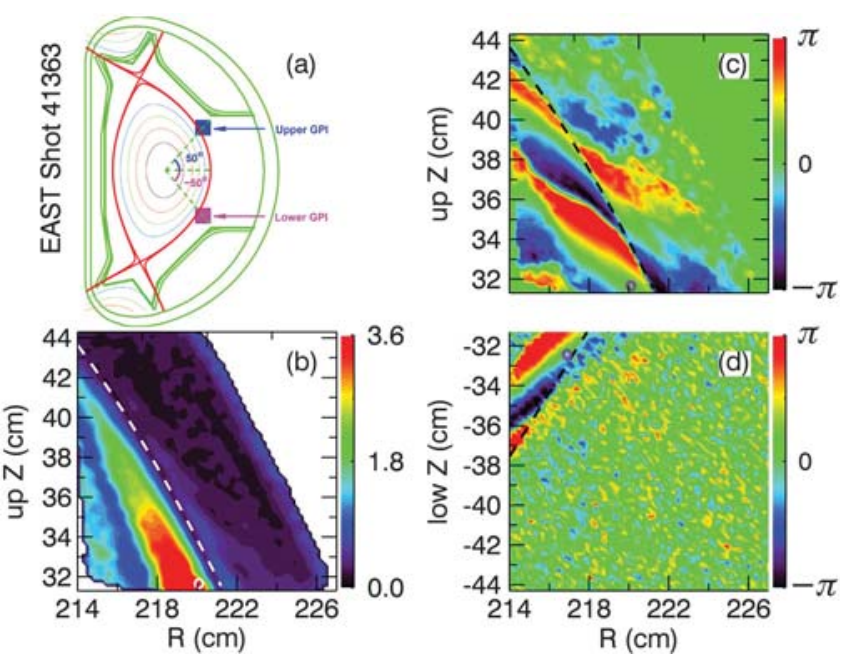

FIG. 1 (color). (a) GPI view areas. (b) Spectral cross power of the GPI relative fluctuations, (c) cross phase from the upper view area, (d) cross phase from the lower view area in the ECM frequency range with respect to a reference pixel as marked by a circle. The dashed lines indicate the locations of the separatrix.

$P_{\mathrm{ICRF}}=0.5-1.5 \mathrm{MW}$, which is $1-3$ times of the low-high transition threshold power. The appearance of the ECM is independent of ICRF or LHCD heating schemes. The associated $\mathrm{H}$-mode regime is either ELM-free or mixed with irregular small ELMs with target heat load $<2 \mathrm{MW} / \mathrm{m}^{2}$, exhibiting a good global energy confinement quality with $H_{98 y 2}=0.8-1$.

The ECM appears to be localized at the steep-gradient pedestal region, peaking at $\sim 1 \mathrm{~cm}$ inside the separatrix with a radial spread of $\sim 1 \mathrm{~cm}$. Figure 1(b) shows the spectral cross power in the ECM frequency range with respect to a reference pixel. The data are taken from shot 41363 with the double null magnetic configuration [Fig. 1(a)] during an ELM-free H-mode period, averaged over $20 \mathrm{~ms}$, i.e., 6.7-6.72 s, as shown in Fig. 2.

The ECM exhibits a strongly tilted structure in the plane perpendicular to the local magnetic field lines as shown by the cross-phase images in Figs. 1(c) and 1(d). The tilting angles are in the opposite directions for the upper and lower view areas, being nearly up-down symmetrical. This may be induced by magnetic shear [18], since flow shear would tilt the structures in the same direction. In addition, the poloidal wavelength of the ECM is estimated to be $\lambda_{\theta} \sim 8 \mathrm{~cm}$, corresponding to a poloidal mode number $m>50$. Its toroidal mode number is $n=16-19$, obtained from the fast Mirnov coils at the low-field side (edge safety factor $\left.q_{95}=4.7\right)$. The associated magnetic perturbations are not seen at the high-field side.

The ECM is a predominantly electrostatic mode. Its magnetic component is small with $\delta B \sim 0.2 \mathrm{G}$, as detected by small magnetic coils mounted on the reciprocating probe at the ECM location. This corresponds to $\delta B / B_{p} \sim 1 \times$ $10^{-4}$, which is smaller than that of the quasicoherent

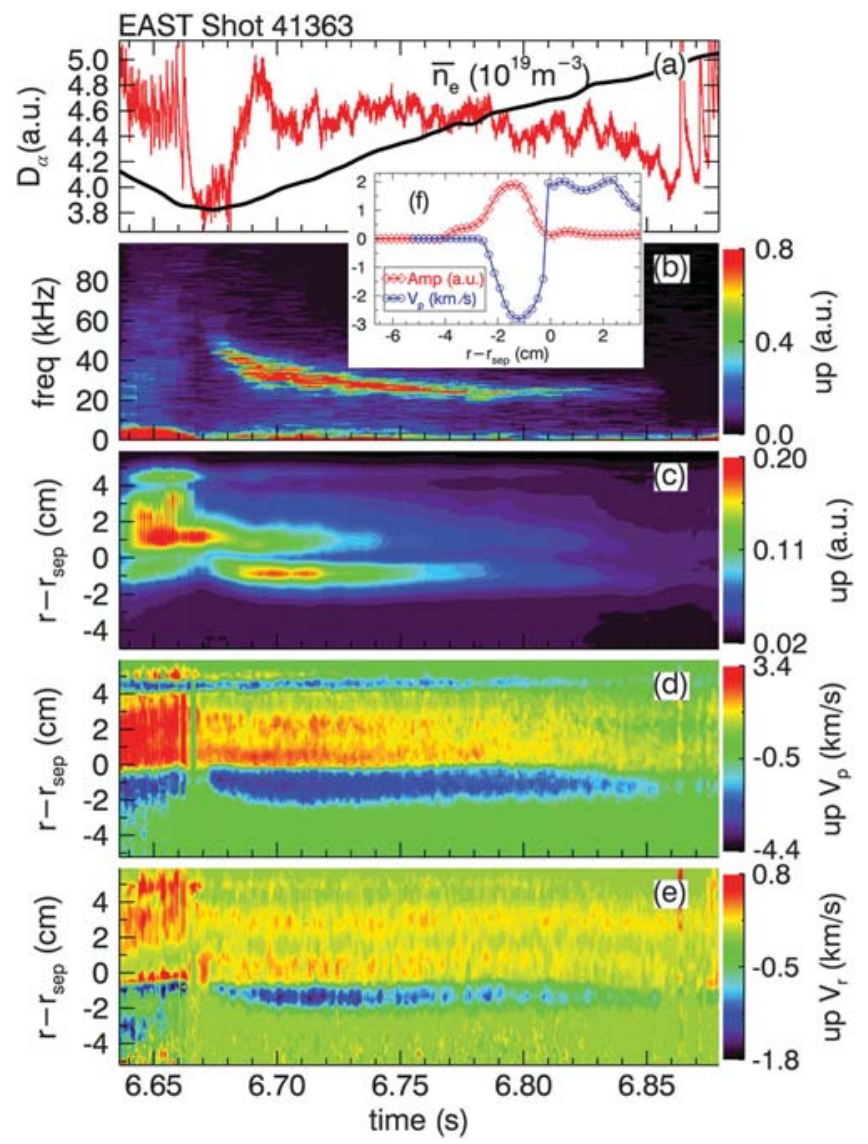

FIG. 2 (color). Time evolution of (a) divertor $D_{\alpha}$ emission (red) and central-line-averaged density (black), (b) power spectrum of the GPI fluctuation inside the separatrix, (c) radial distribution of spectral power in the ECM frequency range, (d) poloidal and (e) radial phase velocity profiles measured by the upper GPI, (f) radial profiles of the poloidal phase velocity (blue), and the ECM relative fluctuation level (red) at $6.71 \mathrm{~s}$.

mode in the C-Mod EDA H mode by one order of magnitude [19]. The magnetic perturbations can be detected by the fast Mirnov coils localized at the low-field side only when the plasma boundary moves close to the outer wall.

The ECM usually starts to appear during pedestal buildup following a low-high transition, or transition from ELMy to ELM-free phase, as shown in Fig. 2. The divertor $D_{\alpha}$ emission level is significantly enhanced [Fig. 2(a)] when the ECM appears, suggesting that the particle transport and divertor recycling are promoted by the ECM. The rising density shown here is largely due to excessive helium gas puffing from the GPI system to facilitate the measurements. In fact, the density is well controlled in such H-mode plasmas without the GPI helium puffing $[11,12]$. The mode usually exhibits an initial frequency chirping-down phase of several tens of milliseconds during the pedestal buildup, as shown in Fig. 2(b). The observed magnitude and direction of the frequency change are in reasonable agreement with a Doppler shift introduced by the change of edge toroidal rotation. The ECM poloidal 
wavelength changes little during this phase. However, the edge toroidal rotation exhibits an increase in the cocurrent direction by $\Delta V_{t} \sim 20 \mathrm{~km} / \mathrm{s}$, which introduces a Doppler shift of $n \Delta V_{t} /\left(2 \pi R_{0}\right) \sim 30 \mathrm{kHz}$, as indicated by the rotation measurements of lithium and carbon impurities from a tangential passive spectroscopy. In contrast, as shown later in this Letter, the ECM propagates in the electron diamagnetic direction. Therefore, the cocurrent rotation increments will downshift the mode frequency by $\sim 30 \mathrm{kHz}$, which is consistent with the observations. In addition, the mode appears to be highly coherent during the initial chirping-down phase, but its spectrum is usually slightly broadened in the following stationary phase.

Figure 2(c) shows the radial distribution of spectral power in the ECM frequency range. The mode is confined in a narrow radial region just inside the separatrix. However, finite spectral power in the same frequency range is also seen in the scrape-off layer (SOL) near the separatrix. In the GPI images, one can clearly see bloblike structures generated by the ECM and moving outwards into the SOL along the tilted patterns [Fig. 1(c)]. This may drive convective transport across the separatrix and promotes the SOL transport, leading to the observed enhanced recycling and $D_{\alpha}$ emissions.

Figures 2(d) and 2(e) show the radial profiles of the poloidal and radial phase velocities, respectively, calculated from the upper GPI fluctuations based on a modified timedelay estimation technique [20]. The ECM propagates in the electron diamagnetic direction inside the separatrix. The fluctuations in the SOL propagate poloidally in the ion diamagnetic direction and radially outwards. There is a sharp velocity shear near the separatrix. The poloidal velocity, $V_{p}$, profile exhibits a $U$ shape structure of $2 \mathrm{~cm}$ in width right inside the separatrix, as shown in the inset plot (f). The ECM appears to be confined in the $U$ shape structure. In the radial direction, the ECM has an inward phase velocity in the upper GPI [Fig. 2(e)] and an outward phase velocity in the lower GPI, which is mainly induced by the poloidal motion of the oppositely tilted ECM structures [Figs. 1(c) and 1(d)].

More information on ECM has been provided by the new diamond-coated four tip graphite probe arrays, installed on both reciprocating probe systems at the outboard midplane, toroidally separated by $89^{\circ}$ [21]. Two tips in the middle of the probe are used as a double probe and the other two tips on either side, poloidally spaced by $\Delta_{p}=7 \mathrm{~mm}$, for floating potential measurements, i.e., $\phi_{f 1}$ and $\phi_{f 2}$, with each tip of $2 \mathrm{~mm}$ in length. This probe array can provide simultaneous measurements of electron density, $n_{e}$, electron temperature, $T_{e}=\left(\phi_{+}-\phi_{f}\right) / \ln 2$, and plasma potential, $\phi_{p}=\phi_{f}+2.8 T_{e}$, where $\phi_{+}$is the potential measured by the positively biased tip and $\phi_{f}=\left(\phi_{f 1}+\phi_{f 2}\right) / 2$.

As shown in Fig. 3, the ECM is detected by one probe array at $\sim 8 \mathrm{~mm}$ and the other at $\sim 3 \mathrm{~mm}$ inside the separatrix during an ELM-free period just following

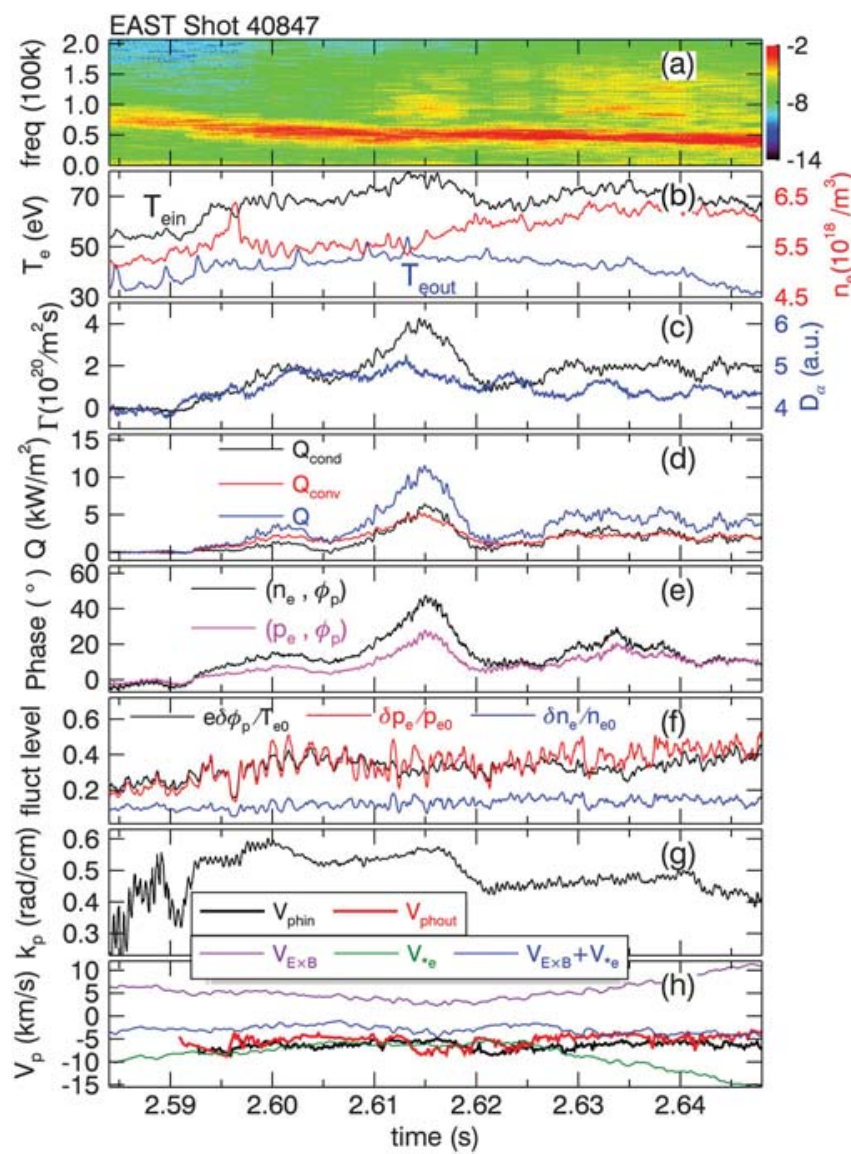

FIG. 3 (color). Time evolution of probe measured (a) crosspower spectrum of electron density and plasma potential fluctuations at $\sim 8 \mathrm{~mm}$ inside the separatrix, (b) electron density (red) and electron temperature $T_{\text {ein }}$ (black) at $\sim 8 \mathrm{~mm}$ and $T_{\text {e out }}$ (blue) at $\sim 3 \mathrm{~mm}$ inside the separatrix, (c) divertor $D_{\alpha}$ emission (blue) and ECM-driven particle flux (black), (d) conductive (black), convective (red), and total heat (blue) fluxes, (e) ECM phase angles between electron density and plasma potential fluctuations (black), between electron pressure and plasma potential fluctuations (pink), (f) relative fluctuation levels of plasma potential (black), electron pressure (red), and electron density (blue), (g) ECM poloidal wavenumber, (h) poloidal phase velocity $V_{\text {ph in }}$ (black) at $\sim 8 \mathrm{~mm}$ and $V_{\text {ph out }}$ (red) at $\sim 3 \mathrm{~mm}$ inside the separatrix, local $\mathbf{E} \times \mathbf{B}$ velocity $V_{E \times B}$ (violet), electron diamagnetic velocity $V_{* e}$ (green), and $V_{E \times B}+V_{* e}$ (blue).

the low-high transition. The mode frequency is near $f_{* e}+f_{E \times B}$, where $f_{* e}=\omega_{* e} / 2 \pi=k_{p} V_{* e} / 2 \pi$ is the electron diamagnetic frequency and $f_{E \times B}$ is the $\mathbf{E} \times \mathbf{B}$ Doppler frequency. Here, $V_{* e}=\partial_{r} p_{e} /\left(e B n_{e}\right)$ is the electron diamagnetic velocity. Furthermore, a statistical analysis shows that the mode frequency in the stationary state scales roughly with $I_{p}^{2} / \bar{n}_{e}$, but depends weakly on the local MHD ballooning parameter $\alpha_{\mathrm{MHD}}=2 \mu_{0} B^{-2} q^{2} R \partial_{r} p_{e}$, which is consistent with the $\omega_{* e}$ dependence [22].

Figure 3(h) shows the poloidal phase velocities, $V_{\mathrm{ph}}$ in and $V_{\text {ph out }}$, the local electron diamagnetic velocity, $V_{* e}$, the $\mathbf{E} \times \mathbf{B}$ velocity, $V_{E \times B}$, and $V_{E \times B}+V_{* e}$, determined by the 
two probe arrays, where the negative sign corresponds to the electron diamagnetic direction. The radial electric field, $E_{r}$, is calculated from the radial difference of the plasma potential measured by the probes. Both $V_{\mathrm{ph}}$ in and $V_{\mathrm{ph} \text { out }}$ are very close to $V_{E \times B}+V_{* e}$, suggesting that the mode propagates in the electron diamagnetic direction in the plasma frame at a phase velocity near $V_{* e}$. The ECM exhibits a very strong electrostatic nature with the relative fluctuation level of the plasma potential and electron pressure, $e \delta \phi_{p} / T_{e 0} \sim \delta p_{e} / p_{e 0} \sim 0.4$, rising during the initial frequency chirping-down phase as shown in Fig. 3(f). The poloidal wavenumber of the mode estimated from the poloidally separated floating potential measurements is $k_{p} \sim 0.5 \mathrm{rad} / \mathrm{cm}$, as shown in Fig. 3(g), corresponding to a poloidal wavelength of $\lambda_{\theta} \sim 12 \mathrm{~cm}$ at the outboard midplane, similar to that from GPI as aforementioned.

Clear evidence of particle and heat exhaust by the ECM has been obtained from a close examination of the phase relations among the fluctuations. The plasma potential fluctuation associated with the ECM lags behind the electron pressure fluctuation by a phase angle of $\alpha \sim 10^{\circ}$ and the electron density fluctuation by $\alpha \sim 20^{\circ}$, as shown in Fig. 3(e). These phase delays lead to radially outward particle and heat fluxes with a $\sin \alpha$ dependence. The particle flux driven by the ECM at the outboard midplane is $\Gamma \equiv\left\langle\delta n_{e} \delta E_{p}\right\rangle / B=2-4 \times 10^{20} \mathrm{~m}^{-2} \mathrm{~s}^{-1}$, as determined by direct probing [Fig. 3(c)]. Here, $\Gamma$ is defined in terms of cross correlation of the density and poloidal electric field fluctuations, with $\delta E_{p}=-i k_{p} \delta \phi_{p}$. Its time evolution generally follows the divertor $D_{\alpha}$ emissions, which provides another evidence for the enhanced particle exhaust and neutral recycling by the ECM. To estimate the surfaceaveraged driven flux based on only one point measurements at the outboard midplane is difficult due to the poloidal asymmetry of the driven flux, which may peak at the outboard midplane for ballooning-type modes. Preliminary particle balance analysis with the assumption of poloidally uniform flux distribution indicates that it is of the same order of magnitude as the total particle flux across the pedestal. When the poloidal asymmetry is taken into account, the driven flux may be within a factor of a few of the total particle flux, but it would be still sufficient for achieving steady state without ELMs [23]. Furthermore, the ECM drives a significant outward heat flux as well, as shown in Fig. 3(d), $Q=Q_{\text {conv }}+Q_{\text {cond }}=5-10 \mathrm{~kW} \mathrm{~m}^{-2}$. Here, $Q_{\text {conv }} \equiv \frac{3}{2} T_{e} \Gamma$ is the convective heat flux, which is comparable to the conductive heat flux, $Q_{\text {cond }} \equiv$ $\left\langle\delta T_{e} \delta E_{p}\right\rangle n_{e} / B$. The total heat exhaust is estimated to be $Q S=0.2-0.4 \mathrm{MW}$, which is about $15 \%-30 \%$ of the loss power, $P_{\text {loss }}$, through the plasma boundary. In addition, the time evolutions of the phases and the fluxes appear to be correlated with the edge $T_{e}$ gradient, i.e., $T_{e \text { in }}-T_{\text {e out }}$, but have no clear correlation with the edge density [Fig. 3(b)], which may suggest that the instability drive is sensitive to the local $T_{e}$ gradient.
Simulations have been performed using the GYRO's eigenvalue solver [24] in a flux tube domain near the peak gradient region of the EAST pedestal, with realistic geometry from the kinetic EFIT reconstruction and full gyrokinetic species (no impurity), taking into account electromagnetic effects, collisions, and equilibrium rotation. The parameters at the peak gradient location are $n_{e}=$ $2.3 \times 10^{19} \mathrm{~m}^{-3}, T_{e}=0.33 \mathrm{keV}$ (measured by Thomson scattering), $q=4.6$, and $\nu_{e}^{*}=1.5$ with the assumption of $T_{i}=0.96 T_{e}$.

The GYRO simulations indicate that the dissipative trapped electron mode (DTEM) $[7,8]$ is the dominant mode in the ECM wavenumber range in the peak gradient region, with characteristics consistent with those of the ECM. The mode propagates in the electron diamagnetic direction at a frequency of $70-80 \mathrm{kHz}$, which is near the local $f_{* e}$ and similar to the observed frequency at the onset of ECMs [e.g., Fig. 3(a)]. The growth rate of this mode peaks in the range of $0.06<k_{p} \rho_{s}<0.1$, consistent with experimental observations, decreasing towards the high $k_{p}$ region. Here, $\rho_{s}=\left(T_{e} m_{D}\right)^{1 / 2} / e B$ is the ion Larmor radius with deuterium mass $m_{D}$. The eigenfunctions of this mode show typical ballooning structures with an even parity in the electrostatic potential $\delta \phi$ and odd parity in the magnetic potential $\delta A_{\|}$. The real and imaginary components of $\delta A_{\|}$ exhibit the same sign of proportionality as expected for TEM. The amplitude of $\delta A_{\|}$is about 30 times smaller than $\delta \phi$, implying that it is a predominantly electrostatic mode, again in agreement with the experimental observations.

Figure 4 shows the calculated results over a broad range of collisionality and $\eta_{e}$, for $k_{p} \rho_{s}=0.1$, where $\eta_{e}=d \ln T_{e} / d \ln n_{e}$. The growth rate of this mode peaks at $\nu_{e}^{*} \sim 2.2$, consistent with the expectation for DTEM,
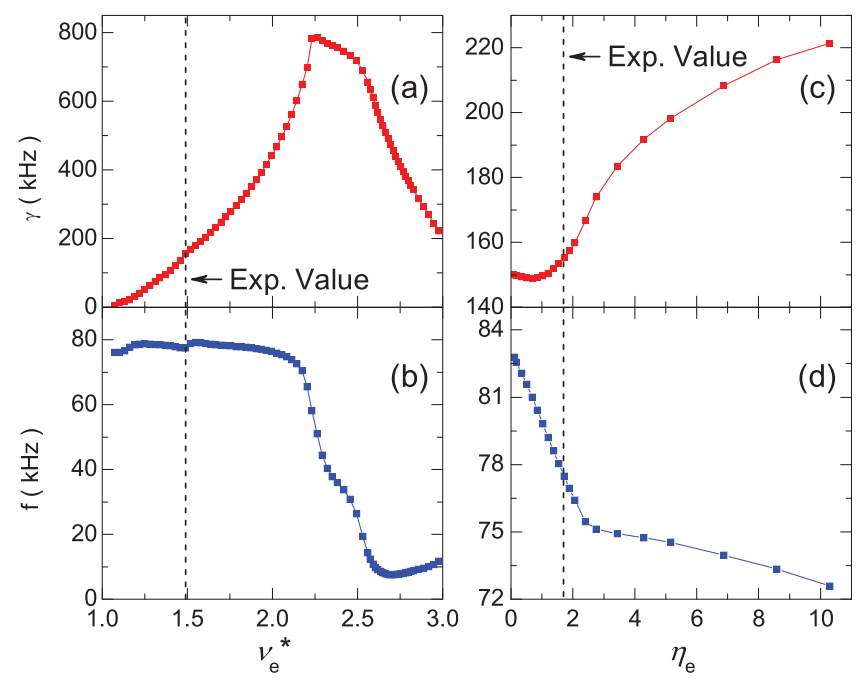

FIG. 4 (color online). (a) Growth rate and (b) real frequency vs normalized collisionality $\nu_{e}^{*}$; (c) growth rate and (d) real frequency vs $\eta_{e}$ of the most unstable mode in the peak gradient pedestal region from the GYRO code simulations. 
which has a maximum growth rate at $\nu_{e}^{*}=\nu_{e i} / \varepsilon \omega_{b e} \sim$ $\omega_{* e} / \omega_{b e}[7,8]$. Here, $\omega_{b e}$ is the thermal electron bounce frequency, $\nu_{e i}$ is the electron-ion collisionality, and $\varepsilon=a / R_{0}$. The collisionality range covers the experimental values in the steep-gradient pedestal region in EAST. Furthermore, the mode appears to be destabilized by the enhanced $T_{e}$ gradient, as seen from the $\eta_{e}$ scan.

In summary, we have discovered a new ECM in the steep-gradient pedestal region of $\mathrm{H}$-mode plasmas in EAST, which exhibits similar characteristics to DTEM, as predicted by the GYRO code simulations. This new ECM provides a channel for continuous particle and heat exhaust across the pedestal, as evidenced by direct probing inside the separatrix, which is essential for the maintenance of long-pulse $\mathrm{H}$-mode plasmas.

This work was supported by National Magnetic Confinement Fusion Science Program of China under Contracts No. 2011GB107001, No. 2011GB101000, No. 2013GB106000, No. 2013GB107003, and No. 2010GB104001, National Natural Science Foundation of China under Contracts No. 10990212, No. 11321092, No. 11075181, the Thousand Talent Plan of China, and the Sino Danish Center for Education and Research. We would like to thank S. J. Zweben, J. Q. Dong, J. Q. Li, and Linjin Zheng for useful discussions. *gsxu@ipp.ac.cn

[1] A. Loarte et al., Plasma Phys. Controlled Fusion 45, 1549 (2003).

[2] A. Mazurenko, M. Porkolab, D. Mossessian, J. Snipes, X. Xu, and W. Nevins, Phys. Rev. Lett. 89, 225004 (2002).

[3] K. Kamiya, N. Oyama, T. Ido, M. Bakhtiari, and JFT-2M Group, Phys. Plasmas 13, 032507 (2006).
[4] K. H. Burrell, T. Osborne, P. Snyder, W. West, M. Fenstermacher, R. Groebner, P. Gohil, A. Leonard, and W. Solomon, Phys. Rev. Lett. 102, 155003 (2009).

[5] E. R. Solano et al. Phys. Rev. Lett. 104, 185003 (2010).

[6] T. E. Evans et al., Phys. Rev. Lett. 92, 235003 (2004).

[7] B. B. Kadomtsev and O. P. Pogutse, in Reviews of Plasma Physics, edited by M. A. Leontovich (Consultants Bureau, New York, 1970), Vol. 5.

[8] C. S. Liu, M. N. Rosenbluth, and W. M. Tang, Phys. Fluids 19, 1040 (1976).

[9] D. R. Ernst et al., Phys. Plasmas 11, 2637 (2004).

[10] C. Angioni, A. G. Peeters, X. Garbet, A. Manini, F. Ryter, and ASDEX Upgrade Team, Nucl. Fusion 44, 827 (2004).

[11] J. Li et al., Nat. Phys. 9, 817 (2013).

[12] B. N. Wan, J. Li, H. Guo, Y. Liang, and G. Xu, Nucl. Fusion 53, 104006 (2013).

[13] R. Schrittwieser et al., in 40th EPS, EPS Conference on Plasma Physics, Espoo, Finland, 2013, P6.017, http://ocs .ciemat.es/EPS2013PAP/pdf/P6.017.pdf.

[14] H. Q. Wang et al. (to be published).

[15] D. L. Rudakov et al., Plasma Phys. Controlled Fusion 44, 717 (2002).

[16] S. C. Liu et al., Rev. Sci. Instrum. 83, 123506 (2012).

[17] O. Sauter, C. Angioni, and Y. R. Lin-Liu, Phys. Plasmas 6, 2834 (1999).

[18] N. Fedorczak, P. H. Diamond, G. Tynan, and P. Manz, Nucl. Fusion 52, 103013 (2012).

[19] J. Snipes, B. LaBombard, M. Greenwald, I. H. Hutchinson, J. Irby, Y. Lin, A. Mazurenko, and M. Porkolab, Plasma Phys. Controlled Fusion 43, L23 (2001).

[20] N. Fedorczak, P. Manz, S. C. Thakur, M. Xu, G. R. Tynan, G. S. Xu, and S. C. Liu, Phys. Plasmas 19, 122302 (2012).

[21] W. Zhang et al., Rev. Sci. Instrum. 81, 113501 (2010).

[22] B. N. Rogers, J. F. Drake, A. Zeiler, Phys. Rev. Lett. 81, 4396 (1998).

[23] G. D. Porter, T. A. Casper, and J. M. Moller, Phys. Plasmas 8, 5140 (2001).

[24] E. A. Belli and J. Candy, Phys. Plasmas 17, 112314 (2010). 